\title{
Infrared short-circuit detection for electrolytic copper refining
}

\author{
R.M. Jia ${ }^{\mathrm{a}}$, X.L. Ma and W.Q. He \\ School of Electronic Information Engineering, rth China of University of Technology, 5 Jinyuanzhuang Road, 100144 \\ Beijing, China
}

\begin{abstract}
This paper proposes an automatic detection method for short-circuit fault that based on the thermal radiation principle of infrared image. During copper electrolytic refining, short circuits between cathode and anode plates will lower the production efficiency. It is necessary to detect short circuits timely to reduce the electricity loss. Firstly, the positive and negative samples were collected that came from the infrared images segmentation of the electrolytic tank images. Then, pixel ordering PCA feature extraction algorithm is proposed to obtain the samples feature. Finally, SVM classifier is used to recognize the short circuits. Experiment results prove that the recognition rate based on proposed method is better than other algorithms, and this method has been applied in the electrolytic copper factory.
\end{abstract}

Keywords: infrared images; short-circuit fault; copper electrolytic; pixel ordering PCA; SVM.

\section{Introduction}

At present, the acquisition of refined copper mainly uses the method of electrolysis. Due to the electrolytic process conditions, the impurities level of electrolyte, the current density unbalance, the quality of anode and cathode plates and so on, cathode copper is easy to appear irregular shape copper knot, which will lead to short-circuit between the positive plate and negative plate. Short circuits between the poles will make the current between cathode and anode increase sharply. As a result of, the power consumption increases and the metal output decreases sharply [1-3]. Thus it is important to detect the short circuits effectively and reduce the economic cost and increase the output.

Currently, the mainly detect methods of short circuits including Reed Switch hand type check slot device and Hall sensor hand type check slot device. The former is simple, but it costs a lot of manpower and it is easy to make mistakes[4]. The latter has a longer service life and is not easy to be damaged, but the effect of detection for the traditional process of electrolytic tank is poor. There are also scholars proposed to establish the function relationship between the infrared image pixel value and the current function of cathode-anode plate to detect the short circuit[5]. However, considering the actual electrolytic process that the electrolytic tanks were covered with insulation cloth, this method is not ideal.

In view of the above mentioned problems, this paper proposes a method, which is based on the infrared radiation feature classification and recognition, to detect the short circuits of the electrode plates. The rest of this paper is organized as follows. Section 2 presents the infrared images of the electrolytic tank which are collected by infrared camera, and split them to a single electrolytic tank image. The method that uses statistical methods to obtain the optimal width of the infrared image

\footnotetext{
${ }^{a}$ Corresponding author : jamin_han@163.com
} 
samples of cathode-anode plate is introduced in section 3. Presented in section 4 is the proposed algorithm that a PCA feature extraction algorithm based on pixel ordering. Data analysis is followed in Section 5. Analysis result demonstrates the effectiveness of the proposed approach. Finally, conclusions are given in Section 6.

\section{Infrared image capture and segmentation of the electrolytic tank}

During the process of electrolysis, a trolley equipped with infrared camera patrols right above the electrolytic tanks to capture the images. And the images will be uploaded to central control room via wireless network to process[6].

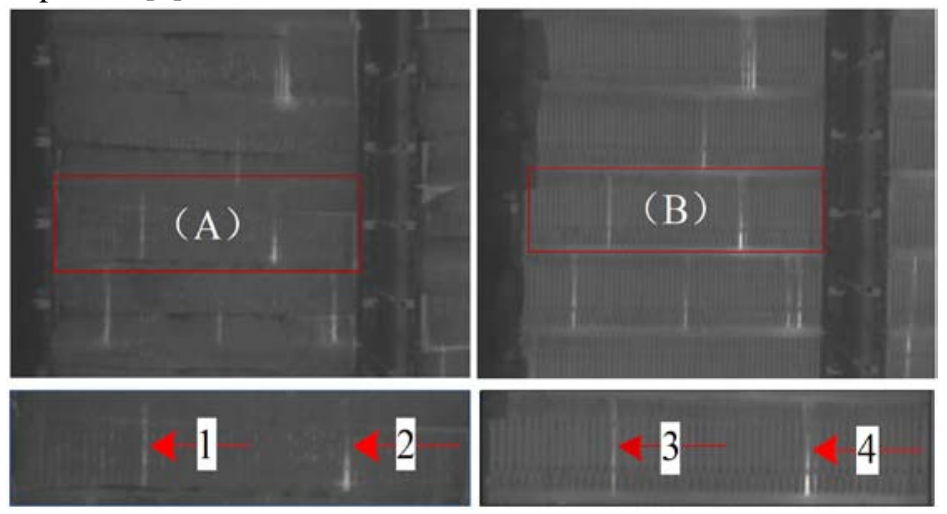

Figure 1. (a) Original image with cloth (b) Original image without cloth (c) electrolytic tanks segmentation image with cloth (d) electrolytic tanks segmentation image without cloth

Electrolytic tank images with cloth and without cloth are shown in Figure 1, which are taken almost at the same time. In the collection of each image contains three electrolytic tanks, and we need to split them into a single cell image. Figure 1 (c) and (d) are the results of the segmentation image of the electrolytic tanks, respectively correspond to the image in the box A and B which are collected in Figure 1 (a) and (b). The arrows 1 4 that refer to the long strips of bright represent the short circuits fault which have been confirmed by artificial and need to be detected.

\section{Sample collection of cathode-anode pairs}

We collected the cathode-anode pairs of the segmented electrolytic tank image, so as to extract the features of the normal electrode bars (positive samples) and the short circuits (negative samples).

Figure 2 shows the working principle of cathode-anode in the process of electrolysis. Figure 2 (a) shows the normal work principle of the electrodes, and figure 2 (b) shows the work principle of shortcircuit.

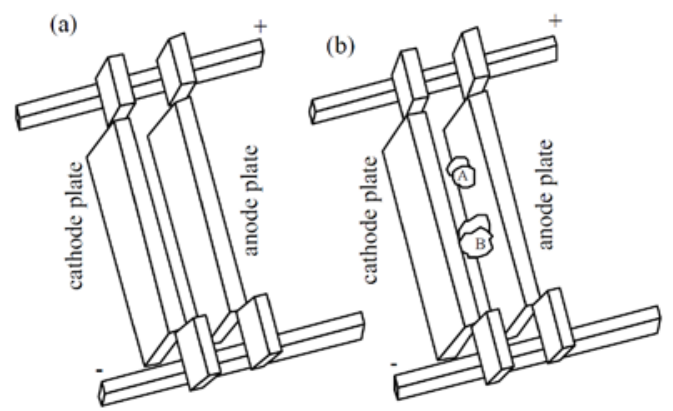

Figure 2. Working principle diagram of cathode-anode (a) normal work principle of the cathode-anode (b) work principle of short-circuit 
In the process of copper electrolysis, the cathode is easy to produce deposited copper. In Figure 2(b), when the deposited copper gradually increased from A to B, it will lead to a short circuit of cathode and anode[7]. At then, the cathode temperature will rise sharply and present bright in the infrared images. In the process of electrolysis, the cathode and anode are arranged in parallel, so the accumulated column value of pixel of the short-circuit electrode plates are higher than the normal electrode plates.

\subsection{Accumulated column pixel value of electrolytic tank}

The sum of column pixels value in the segmented images is calculated to find the peaks which usually are short circuit bars' position. It is given through equation (1):

$$
S_{N}=\left(\sum_{i=l, j=l}^{i=M} G_{i 1}, \sum_{i=1, j=2}^{i=M} G_{i 2}, \ldots, \sum_{i=1, j=N}^{i=M} G_{i N}\right)
$$

Where $\mathrm{M}$ and $\mathrm{N}$ stand for the row and column, $G_{i j}$ represents the gray value of the row $\mathrm{i}$ and column j. Figure 3 shows the pixel column accumulation curves of electrolytic tanks.

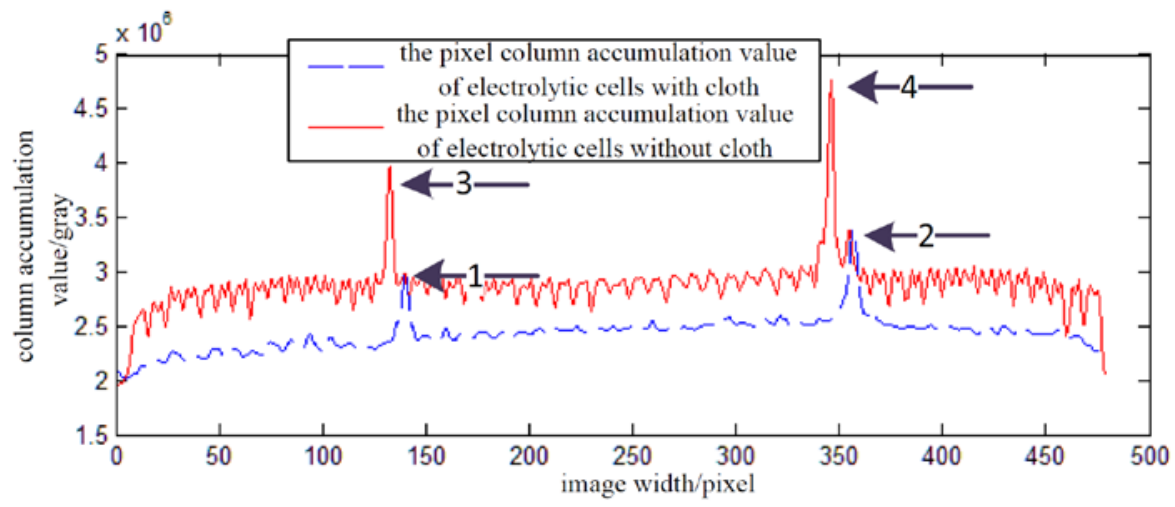

Figure 3. The pixel column accumulation value curve of electrolytic tanks

The experimental results show that the cumulative value of the column about the short circuits cathode will be a severe wave peak (local maximum), but the normal cathode-anode and the shortcircuit anode may not have the crest. As we can see Figure 3, the column accumulation value of the short circuits cathode plates in the electrolytic tank images with a cloth (like 1 and 2) is similar to the normal cathode-anode electrode plates without cloth. In the actual production process we were unable to determine the electrolytic tanks whether cover with the cloth by the shooting images. Thus, it is difficult to establish the temperature change model of the electrolytic tank images which with the cloth. Therefore, we need to collect samples of a variety of situations, such as cloth and without cloth, to extract the stable features of the samples.

\subsection{The selection method of samples middle column reference}

The short-circuit cathode has a higher temperature and the image of the column value will have a peak, but the temperature between the short-circuit anode and the normal cathode-anode pairs is very small. So, we can locate the short circuits electrode plates by detecting the short-circuit cathode. In order to ensure that the collected samples contain enough useful information, the wave crest is chosen as the baseline of the middle of the samples. Figure 4 shows the selection principle of negative samples.

In figure 4, the white boxes represent the cathode and the grey box indicates the anode. The red dashed line boxes represent the cathode-anode pairs, and blue lines represent the middle column of the 
cathode plate. We choose the black line box as the negative sample, which is based on the blue line 1 as the centre line.
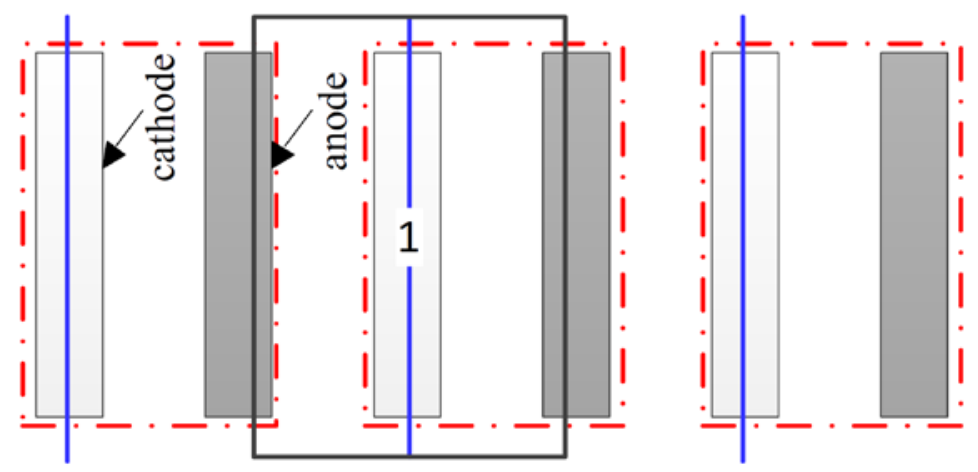

Figure 4. The selection principle diagram of negative samples

In this paper, the width of the segmented image is 480 pixels, containing 53 pairs of cathode-anode plates, the average width of each cathode-anode pair is about 9.06 pixels and the average width between the two adjacent cathode plates is about 7 pixels. The method for selecting the width of positive sample is to take the position of the non-short circuit wave crest as the middle column and extend it to a certain scope. The non-short circuit wave crest of positive samples in the neighbourhood of 21 pixels is the maximum. However, with an average width as the sample width is not the best. Therefore, the optimal width of the sample is calculated by using statistical method.

\subsection{Optimal sample width}

In the following, the optimal width of the sample is given by using the method of statistics. The superposition graph is defined by equation (2):

$$
S_{n}=\sum_{k=1}^{k=n} G_{i j} / n
$$

Where $G_{i j}$ stand for the gray value of the row $\mathrm{i}$ and column $\mathrm{j}, \mathrm{n}$ is the total number of images, and $S_{n}$ is a superimposed image.

In this paper, we collected 5000 normal samples and 5000 short-circuit samples. Then the superposition images of 5000 normal samples and 5000 short-circuit samples were calculated. Figure 5 (a) and 5 (b) shows the superimposed image of the normal samples and short-circuit samples. Their cumulative value curves are shown in Figure 5 (c).
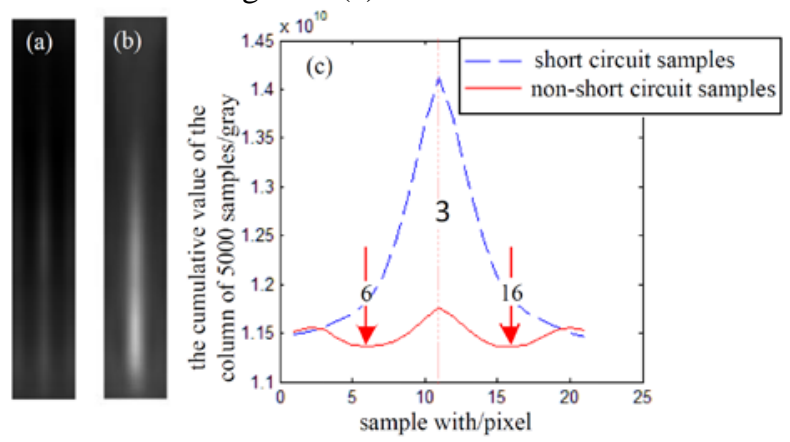

Figure 5. Sample superimposed image and column accumulation value curve: (a) non-short circuit sample superimposed image (b) short circuit sample superimposed image (c) cumulative value curves of sample superimposed image 
In the figure 5(c), the red line has three peaks which represent three normal cathode plates. In the choice of the samples with the largest peak as the reference centre, so the middle of the peak is larger than either of the two sides. Arrow 6 and arrow 16 represent line trough, they also represent the horizontal coordinate. The dotted line has only one peak. It is because of the high temperature of the short-circuit electrode plates and radiation to both sides, so that the temperature of the cathode plate on both sides is greatly affected and the small peaks on both sides disappeared.

In this paper, the sample width is selected from 6 to 16 as the final sample width, that is to say the optimal sample width is 11 . The selection of the width fully guarantees the temperature information of the short-circuit cathode plates. We select the dotted line 3 in the figure 5 as the centre line, as much as possible outward expansion, while ensuring the characteristic information of the normal cathode plate. In addition, we show the correctness of the best sample width by comparing the optimum sample width and the other sample width.

\section{Sample feature extraction}

At present, the common image features are gray statistical features, texture edge feature, image algebra feature and image transform coefficient feature. So, we analyze the applicability of the mean, variance, histogram, LBP (Local Binary Patterns), PCA (Principal Component Analysis), which are used in the detection of short circuit plates. In this paper, the method is to order the sample image pixels, and then extract the PCA features. Finally, identify the short circuits by the obtained feature. The experimental results show that this method is better than the traditional methods.

\subsection{PCA feature}

Principal component analysis belongs to the category of feature extraction. The principle is to find the most original data to represent the projection direction in the condition of least mean square, and high dimensional will be mapped to low dimensional, while retaining the main features[7]. The PCA steps for a pair of images are as follows:

The image were regarded as a matrix of $M$ rows and $N$ columns, then arrange it in a onedimensional vector $\boldsymbol{\eta}$ with a size of $M^{\prime} N$. It is given through equation (3):

$$
\boldsymbol{\eta}=\left[x_{1}, x_{2}, \cdots, x_{M \times N}\right]
$$

Where $x_{i}$ stand for the vector value of the ith position.

The matrix generated by PCA transform can be both the total covariance matrix and the covariance matrix between classes. Considering the category of this paper is less and the number of samples of a single category is more, we select the total covariance matrix as a generating matrix. The class covariance matrix of the samples can be expressed by equation (4):

$$
S=\frac{1}{L} \sum_{i=1}^{L}\left(\xi_{\mathrm{i}}-\bar{\xi}\right)\left(\xi_{\mathrm{i}}-\bar{\xi}\right)^{T}
$$

Where $\xi_{i}$ represents the ith row vector, $\bar{\xi}$ represents the mean vector of the population samples.

Then, the eigenvalue decomposition is performed on the $S$, and the characteristic value ${ }_{i}$ and the normalized feature vector $\mathbf{e}_{\mathbf{i}}$ are obtained. We arrange the feature vectors according to the size of the feature vector from the large to small, that is $\lambda_{1}>\lambda_{2}>\cdots>\lambda_{Q}$. The larger of the characteristic value, the larger difference between the two classes. For the most of the information is contained in the feature vector corresponding to the $5 \% \sim 10 \%$ eigenvalue[8]. In this paper, we select the first 50 dimensions of the PCA transform as the feature vector. The projection matrix, which is composed by feature vectors corresponding to the largest eigenvalues of the former p, can be expressed as the equation (5): 


$$
\mathbf{W}=\left[\mathbf{e}_{1}, \mathbf{e}_{2}, \cdots, \mathbf{e}_{\mathbf{p}}\right]
$$

Where $\mathbf{W}$ stand for the projection matrix. Finally, by using the projection matrix, we obtain the transform characteristic vector of the sample images. It is can be showed by the equation (6):

$$
\mathbf{Y}=\mathbf{W}^{T} \xi
$$

PCA reflects the main feature of the images. In addition, it not only reflects the direction of the principal component, but also reflects the strength of the principal component. Experiments extract the PCA features of the samples with short circuit electrode plates and non-short circuit electrode plates. Figure 6 shows the PCA features of the samples.
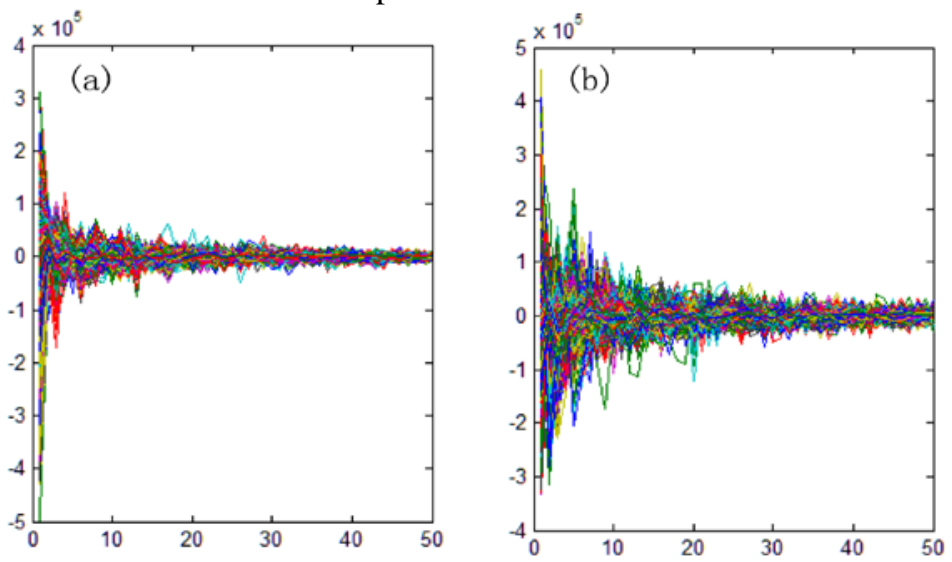

Figure 6. PCA feature images: (a) PCA feature of the short circuit plates (b) PCA feature of the non-short circuit plates

From Figure 6, we can see that there is a clear difference between the short circuits and non-short circuit. They have the similarity in the principal component direction but the difference is larger in the principal component strength, which can be better distinguished them.

\subsection{The PCA feature of image pixel ordering}

Ordering is defined as the process by which all elements are arranged according to a particular logical law. For the image, it is according to the pixel value rearranged from large to small, and gets a new one. Figure 7 shows the results of a short circuit plate after pixel ordering.

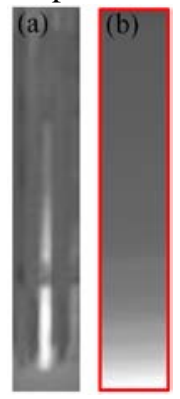

Figure 7. Image pixel ordering: (a) the original image of short circuit plate (b) the pixel ordering image of short circuit plate

The purpose of image pixel ordering is to increase the similarity of similar samples and reduce the similarity of different samples. In order to improve the recognition rate, in this paper, after the 
samples image pixels ordering, the PCA feature is obtained. The results of experiment are given in Figure 8:
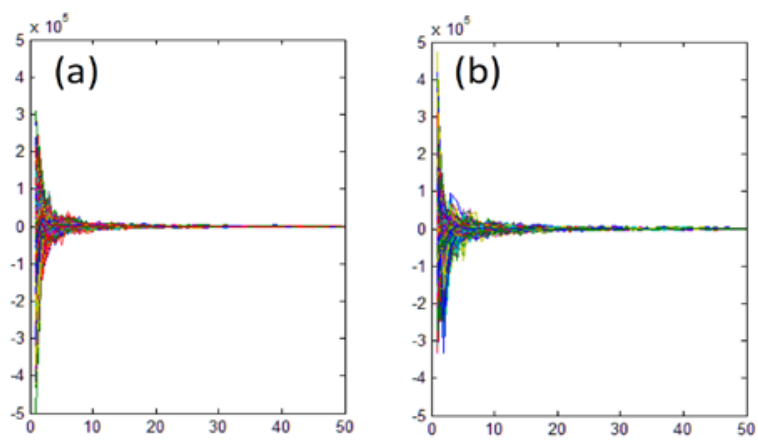

Figure 8. PCA feature after pixels ordering: (a) PCA feature of the short-circuit electrode plates after pixels ordering (b) PCA feature of the normal electrode plates after pixels ordering

From the experimental results in Figure 8, we can see that the similar samples are more similar, the characteristic curve will be more consistent. A comparison between Figure 6 and figure 8 can be seen that the characteristic curve in Figure 8 is more consistent than that of the characteristic curve in Figure 6.

\section{Experimental results}

Through the experiments above, we get the feature vector of the samples, and need to use the classifier to classify the feature, so as to correctly judge the position of short circuits.

In this paper, k nearest neighbor (k-NN) [9] and support vector machine (SVM) [10]are selected as the classifiers. In order to prove the correctness of the method proposed in this paper, we select the gray histogram feature, local two value model (LBP) as contrast. In the 5000 positive and negative samples, we choose 2500 as the training, the remaining 2500 as a test. Finally, we get the recognition rate using different feature algorithm. Table 1 shows the recognition:

Table 1. Recognition rate and computation time of different feature algorithm.

\begin{tabular}{ccc}
\hline algorithm of & \multicolumn{2}{c}{ recognition rate } \\
\cline { 2 - 3 } feature extraction & NNA & SVM \\
\hline gray histogram & $71.2 \%$ & $79.6 \%$ \\
LBP & $84.1 \%$ & $85.6 \%$ \\
PCA & $93.5 \%$ & $97.5 \%$ \\
pixel ordering PCA & $98.3 \%$ & $98.8 \%$ \\
\hline
\end{tabular}

The recognition rate of sample width from 1 to 21 is given in Figure 9:

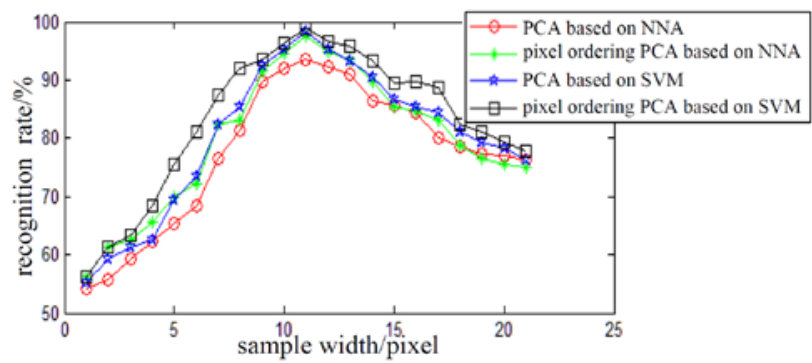

Figure 9. Recognition rate curve of PCA and pixel ordering PCA algorithms under different classifiers. 
It is seen from Figure 9, when the sample width is 11, the recognition rate is the highest. The results show that the optimum sample width is correct.

\section{Conclusions}

In this paper, a method based on the thermal radiation principle of infrared image is proposed to detect the short circuits in the electrolytic tanks. An optimal selection scheme of samples is given under the conditions with a dense distribution of the electrodes and high temperature effect of short circuits. Then the pixel ordering PCA feature extraction method is proposed, according to the PCA feature, which can effectively improve the recognition rate of the short circuits. Experimental results show that, under the same condition of the classifier, the short circuits recognition rate of the pixel ordering PCA algorithm, which can reach above 98\%, is higher than that of the traditional PCA algorithm, lBP algorithm and gray histogram algorithm.

The detection method proposed in this paper is economical and applicable, and it is also safe and reliable. Not only reduces the risk of acid liquid corrosion, but also can quickly and timely detect the short circuits and guide the workers to remove. Finally, the output of copper is increased and the energy loss is reduced. The method is also applicable to other metals, such as nickel and cobalt, which are used in metallurgy.

\section{References}

1. E.P.Wiechmann, P.E. Aqueveque, R.P. Burgos, IAS Annual Meeting, 2074(2007)

2. X.Zhao, Nonferrous Metals Engineering \& Research, 29, 12(2008)

3. E.P.Wiechmann, G.A.Vidal, A.J. Pagliero, IEEE Transactions on Industry Applications, 2, 851(2006)

4. W.l. Zhang, J. li, Energy Saving Of Non-Ferrous Metallurgy, 4, 29(2006)

5. R.T. Zhao, Y. Zhang, D. H. li, CIESC Journal, 66, 1807,(2015)

6. X.Y. Zhang, H. Gong, Q. Qi, Electronic Design Engineering, 23, 183(2015)

7. X.S.liu,Computer \& Digital Engineering, 39, 125(2011)

8. C.D.Tong, X.H. Xu, CIESC Journal, 66, 4103(2015)

9. F. lü, N. Du, C. l. Wen, Acta Electronica Sinica, 40, 2391(2012)

10. Q.Chen, J.Tian, H.N. Huang, C.H. Zhang, Chinese Journal of Scientific Instrument, 34,1416(2013) 